Methods Different forms of bLf (iron-depleted; apo-bLf, ironsaturated; holo-bLf, and manganese-saturated; Mn-bLF) and BSA-FITC were encapsulated in calcium-alginate and chitosancoated alginate particles. Microgel particles were fabricated using the emulsification/internal gelation method. Protein encapsulation efficiency was confirmed by fluorescence microscopy imaging of BSA-FITC-loaded hydrogel particles. In vitro release studies conducted in $\mathrm{pH}$-simulated gastrointestinal conditions were employed to investigate protein-loading efficiency and release rate of encapsulated protein. The various encapsulated bLf forms were evaluated for their influence on intestinal epithelial barrier function and cell viability alone, and in combination with purified whole $C$. difficile toxins $\mathrm{A}$ and $\mathrm{B}$ or bacterial supernatant samples of the epidemic $027 \mathrm{C}$. difficile strain. Enterocyte viability and epithelial permeability were assessed using trypan blue exclusion, MTT cytotoxicity assay and changes in trans-epithelial electrical resistance (TEER) in Caco-2 cells, respectively.

Results Alginate microparticles are suitable for encapsulation and $\mathrm{pH}$-triggered release of metal-bound bLF proteins. The application of bLf $(5 \mathrm{mg} / \mathrm{mL})$ delivered from alginate microparticles to human intestinal epithelial cells (hIECs) significantly reduced the cytotoxic effect of toxin $\mathrm{A}$ and bacterial supernatant samples on Caco- 2 cells, as illustrated by increased TEER values and enhanced Caco- 2 cell viability. Pre-treatment of Vero cell monolayers with all forms of encapsulated bLf followed by exposure to toxin B or bacterial supernatant induced a fall in mitochondrial enzyme activity.

Conclusions Our results are the first to suggest that alginatebLf microparticles show protective effects against C. difficile toxin-mediated mucosal damage and impairment of barrier function in hIECs. The future potential of lactoferrin-loaded alginate microparticles in the treatment and prevention of CDI deserves further investigation in preclinical studies.

\section{PTU-047 HIGH PREVALENCE OF CLOSTRIDIUM DIFFICILE RIBOTYPE 078 IN IBD OUTPATIENTS}

\begin{abstract}
${ }^{1}$ Tanya Monaghan*, ${ }^{2}$ Tim Sloan, ${ }^{3}$ Jane Freeman, ${ }^{4}$ David Eyre, ${ }^{1}$ Melanie Lingaya, ${ }^{1}$ Yirga Falcone, ${ }^{1}$ Helen Foreman, ${ }^{1}$ Jill Garrett, ${ }^{1}$ Yash Mahida, ${ }^{1}$ Nina Lewis, ${ }^{3}$ Mark Wilcox. ${ }^{1}$ NIHR Nottingham Biomedical Research Centre, Nottingham University Teaching Hospitals NHS Trust and University Of Nottingham, Nottingham, UK; ${ }^{2}$ University of Nottingham, Nottingham, UK; ${ }^{3}$ Leeds Teaching Hospitals and University of Leeds, Leeds, UK; ${ }^{4}$ Nuffield Department of Medicine, University of Oxford, Oxford, UK
\end{abstract}

\subsection{6/gutjnl-2018-BSGAbstracts.388}

Background and Objectives Point prevalence studies have reported higher carriage rates of $C$. difficile in IBD patients compared with the general population, but longitudinal prospective data are lacking. The objectives of this observational study were to investigate and molecularly characterise isolates of $C$. difficile, collected prospectively on a monthly basis over a one-year period among IBD outpatients and healthy controls (HC).

Methods At enrolment, recruited participants had established diagnoses of UC $(n=16)$ and Crohn's disease $(n=6)$ and reported no recent hospitalisation or exposure to antibiotics. PCR ribotype and toxin status (cytotoxigenic culture) were determined for all +ive stool cultures. All participants underwent a monthly telephone interview to identify potential risk factors for $C$. difficile acquisition (changes in medications, exposure to antibiotics, clinic attendances, hospitalisation) and to assess for disease activity (Harvey-Bradshaw Index and Simple Clinical Activity Colitis Index).

Results Two patients underwent physician-initiated laboratory testing of C. difficile during the sample collection phase, although no participants developed or were treated for C. difficile infection. C. difficile was cultured from 29/223 samples (13\%) representing $16 / 22$ patients and 1 of 5 HC with concurrent antibiotic exposure in $6 / 29$ visits (20\%). Of the toxin +ive isolates $(\mathrm{n}=25 ; 078,005,302$ and 015), $72 \%$ $(n=21)$ were PCR ribotype 078. Toxigenic negative ribotypes included 023, 026 and 656. Of those toxin +ive isolates, 9 samples (36\%) were associated with relapsing IBD of which $7 /$ 9 were ribotype 078. Multiple stool specimens also tested +ive for different ribotypes in 3 patients with UC, all of whom were taking regular immunosuppressants. WGS studies of the 078 isolates revealed marked genetic similarity, with only 3 of the 21 isolates varying by 1 or more nucleotides when compared to the 078 reference genome, suggesting there may have been a common source for cross-transmission.

Conclusions The high prevalence of PCR ribotype 078 in this IBD outpatient cohort is consistent with the recent emergence of this strain in the community. These results reinforce the importance of testing all in-and outpatients with an apparent flare or relapsing IBD for carriage of toxigenic C. difficile to inform optimal management strategies. Future research is needed to understand the predominance of 078 isolates in IBD, particularly in the context of clinical relapse.

\section{PTU-048 SERUM TRACE METAL CONCENTRATIONS IN CLOSTRIDIUM DIFFICILE INFECTION AND THEIR RELATIONSHIP TO DISEASE SEVERITY}

${ }^{1}$ Tanya Monaghan*, ${ }^{2}$ Tahseen Jilani, ${ }^{3}$ Marcin Frankowski, ${ }^{4}$ Klaudyna Spiewak,
${ }^{4}$ Malgorzata Brindell. ' NIHR Nottingham Digestive Diseases Biomedical Research Centre,
Nottingham University Teaching Hospitals NHS Trust and University Of Nottingham,
Nottingham, UK; ${ }^{2}$ School of Computer Science, Advanced Data Analysis Centre, University
of Nottingham, Nottingham, UK; ${ }^{3}$ Faculty of Chemistry, Adam Mickiewicz University,
Poznan, Poland; ${ }^{4}$ Faculty of Chemistry, Jagiellonian University, Krakow, Poland

\subsection{6/gutjnl-2018-BSGAbstracts.389}

Introduction Trace metals play a central role in host-pathogen interactions, impacting both microbial growth/pathogenicity and antimicrobial host immune defences. The main objective of this study was to determine the relationship between serum trace metal concentrations and severity of $C$. difficile infection (CDI).

Methods We analysed biobanked serum samples previously collected from a single-centre prospective observational study in which we recruited consecutive hospitalised patients $\geq 18$ years with symptomatic toxin-positive $C$. difficile diarrhoea. Severe C. difficile was defined as WCC $\geq 15 \times 10^{9} / \mathrm{L}$ or an increase in serum creatinine $\geq 1.5$ above baseline, or pseudomembranous colitis, megacolon, need for colectomy or septic shock requiring ICU admission. Selected trace metal (iron, selenium, zinc, cadmium, cobalt, copper, magnesium, manganese, nickel and lead) concentrations in sera were determined by inductivelycoupled mass spectrometry (ICP-MS). Mean biometal concentrations were compared between mild and severe CDI cases using Mann-Whitney test. Univariate and multivariate regression analyses were used to identify clinical and biochemical factors associated with risk of severe CDI.

Results A total of 224 serum samples derived from 149 patients [ $\mathrm{n}=65$ male; mean age (S.D.) 65.7 years $(16.4) ; n=84$ 
female; 68.3 years (19.3)] were included. The median concentrations $(\mu \mathrm{g} / \mathrm{L})$ of iron, zinc and selenium were significantly lower in severe CDI cases compared with those with mild infection. Using CART decision tree analysis, 4 variables (peak WCC, selenium, Charlson co-morbidity index and magnesium) were found to be good predictors of severe CDI with sensitivity, specificity, accuracy, positive predictive value, negative predictive value and ROC of $84.8 \%, 98.9 \%, 94.3 \%, 97.5 \%$, $93.1 \%$ and 0.848 , respectively.

Conclusions Novel therapeutic interventions that modulate the availability of trace metals may substantially impact on disease outcomes in CDI. Mechanistic studies are required to establish the source of metals detected and to determine their relevance to oxidative stress, impaired immune response and the promotion of C. difficile bacterial virulence.

\section{PTU-049 RECENT COLORECTAL CANCER INCIDENCE TRENDS: IS OVERDIAGNOSIS A PROBLEM IN SCREENING?}

\footnotetext{
${ }^{1,2}$ Joanne Morling* ${ }^{*}{ }^{1}$ Caroline Chapman, ${ }^{1,2}$ Richard Logan. ${ }^{1}$ Bowel Cancer Screening Programme, Nottingham, UK; ${ }^{2}$ Division of Epidemiology and Public Health, University of Nottingham, Nottingham, UK
}

\subsection{6/gutjnl-2018-BSGAbstracts.390}

Introduction Colorectal cancer (CRC) screening using biennial gFOBT was introduced in England in September 2006. ${ }^{1}$ and by 2010 was being offered $>90 \%$ of $60-69$ year olds, rising to $>95 \%$ of $60-74$ year olds by 2014 . Overdiagnosis of cancer is a major concern of cancer screening programmes; in breast cancer it has been estimated that around 50\% of screen detected cancers might be due to overdiagnosis. This study seeks to examine the trends in CRC incidence and ascertain what impact screening has had on incidence.

Methods Data for the period 2001-16 was extracted from the ONS website (www.ons.gov.uk) and CRC incidence rates by 5 year age bands from age 45 calculated. CRC was defined according to the ICD 10th Revision codes C18 (colon) and C19/C20/C21 (rectum, recto-sigmoid and anus). Changes in incidence rates in age groups never offered screening were compared with those offered screening (age group 60-74 years).

Results As shown in table 1 CRC incidence in the 60-64 age band has increased, peaking at 22\% (M) and 17\% higher $(\mathrm{F})$ in $2009 / 10$ coinciding with the completion of screening rollout before reducing somewhat. In the 65-69 age band there were similar peaks $(21 \% \mathrm{M}$ and $17 \% \mathrm{~F})$ in $2008-10$ before a decline to below pre-screening rates. In the 70-74 age band for whom screening started in 2010 there were peaks in 2011/12 (17\% M and 19\% F) before declining below prescreening rates. These patterns were similar for both colon (C18) and for rectal (C19-21) cancers.

Abstract PTU-049 Table 1 Relative changes in colorectal cancer incidence (C18-21) by 5 year age-band in England \& Wales from 2005 to 2015

\begin{tabular}{llllllllll}
\hline & $45-$ & $50-54$ & $55-59$ & $60-64$ & $65-$ & $70-74$ & $75-79$ & $80-$ & $85+$ \\
& 49 & & & & 69 & & & 84 & \\
\hline Men & $+1 \%$ & $-5 \%$ & $+1 \%$ & $+12 \%$ & $-6 \%$ & $-11 \%$ & $-10 \%$ & $-3 \%$ & $+3 \%$ \\
\multirow{2}{*}{$\begin{array}{l}\text { Women } \\
\end{array}$} & $+15 \%$ & $+20 \%$ & $+18 \%$ & $+14 \%$ & $-4 \%$ & $-4 \%$ & $-4 \%$ & $+5 \%$ & $+8 \%$ \\
& $\%$ & & & & & & & & \\
\hline
\end{tabular}

Conclusion At this point we could find no evidence of over diagnosis of CRC. While there has been a $13 \%$ increase in CRC incidence in the 60-64 age band, consistent with the first (prevalent) screening round there has been no sustained increase in the older age bands offered screening. In contrast there has been an increase in incidence of rectal cancer in women under age 60 .

\section{REFERENCE}

1. Logan RF, Patnick J, et al. Outcomes of the BCSP in England after the first 1 million tests. GUT 2012 0ct;61:1439-46.

\section{PTU-050 THE SUCCESSFUL IMPLEMENTATION OF FAST-TRACK ROUTINE TESTING FOR MICROSATELLITE INSTABILITY IN A COLORECTAL CANCER PATHWAY}

${ }^{1}$ Wee Sing Ngu*, ${ }^{1}$ Sarah Mills, ${ }^{2}$ Harsh Sheth*, ${ }^{2}$ John Burn, ${ }^{1}$ Thomas Lee. ${ }^{1}$ Northumbria Healthcare NHS Foundation Trust, Newcastle Upon Tyne, UK; ${ }^{2}$ Institute of Genetic Medicine, Newcastle University, Newcastle upon Tyne, UK

\subsection{6/gutjnl-2018-BSGAbstracts.391}

Introduction The National Institute for Health and Care guidelines (DG27, Feb 2017) recommend that all patients with colorectal cancer (CRC) should undergo testing for deficient deoxyribonucleic acid (DNA) mismatch repair activity, whose by-product is microsatellite instability (MSI) in DNA. Historically in our trust, MSI testing was done infrequently, in selected high-risk patients, on preserved pathology specimens and with a long wait for results. A new patient care pathway incorporating MSI testing on fresh biopsy tissue with a rapid turnaround time was introduced in January 2017. This service evaluation reviewed performance in the first year of this new pathway.

Methods Endoscopists were asked to send an additional fresh biopsy for MSI assay at endoscopic diagnosis of significant neoplasia from January 2017. Data for all patients newly diagnosed with CRC between 1 st Jan 2017 to 31 st December 2017 were exported from a prospectively populated database. Results A total of 374 patients were identified, median age 72 (range 30-96) of whom 226 (60.4\%) patients were diagnosed at endoscopy. One hundred and ninety-one $(51.1 \%)$ of all patients had MSI assays performed, 142 (62.8\%) of those endoscopically diagnosed. Twelve (6.3\%) of the patients tested were MSI-high. Median time from submission of sample to result was 13 days (range $3-32$ ).

Conclusions Compliance with MSI testing at endoscopic diagnosis is not yet $100 \%$, but this study illustrates that the MSI test can be integrated into the patient care pathway in an NHS setting and used to personalise patient care as turn-around times are sufficiently short for the results to be integrated into pre and post-operative multidisciplinary team meeting discussions.

\section{PTU-051 SHOULD FOBT POSITIVE PATIENTS WITH PREVIOUS LOW RISK IN SCREENING PROGRAM COLONOSCOPY HAVE FURTHER COLONOSCOPY?}

${ }^{1}$ David Nylander*, ${ }^{2}$ Emma Crossland, ${ }^{2}$ Heather Dixon. ${ }^{1}$ Newcastle Upon Tyne NHS Foundation Trust, Newcastle upon Tyne, UK; ${ }^{2}$ North of Tyne Bowel Cancer Screening Centre, Newcastle upon Tyne, UK

\subsection{6/gutjnl-2018-BSGAbstracts.392}

Introduction and aim Current British Society of Gastroenterology guidelines suggest patients who are deemed low risk after 